\title{
Revisando las metas de la tensión arterial
}

\author{
Reviewing the goals of blood pressure
}

María Victoria Salgado*

Salgado MV. Revisando las metas de la tensión arterial. Evid Act Pract Ambul. 2015;18(4):123. Oct-Dic.

En los últimos años, varias guías de práctica clínica cambiaron los objetivos buscados de tensión arterial (TA ${ }^{1-4}$. De acuerdo a estas modificaciones, se propusieron: a) metas más laxas en adultos mayores, a saber, $150 / 90 \mathrm{mmHg}$, por el mayor riesgo de sufrir efectos adversos debidos a la medicación; b) metas en diabéticos similares a las de la población general, es decir, 140/90 $\mathrm{mmHg}$.

El estudio ACCORD (Action to Control Cardiovascular Risk in Diabetes Trial), que asignó pacientes diabéticos a tratamiento intensivo de la TA (objetivo de TAS $<120 \mathrm{mmHg}$ ) vs. estándar (objetivo de TAS $<140$ $\mathrm{mmHg}$ ), no encontró diferencias en la mortalidad por cualquier causa, ni en un resultado compuesto que incluyó infarto agudo de miocardio (IAM) no fatal, accidente cerebrovascular (ACV) no fatal y muertes cardiovasculares.

Asimismo, se flexibilizó el momento de inicio del tratamiento farmacológico en hipertensos grado I sin daño de órgano blanco y con bajo riesgo cardiovascular (CV), planteándose incluso que estos pacientes podrían no requerir tratamiento farmacológico en tanto su riesgo CV no aumentase ${ }^{2}$. En una revisión Cochrane que comparó tratamiento farmacológico vs placebo en hipertensos grado 1 sin enfermedad cardiovascular, el tratamiento no redujo la mortalidad ni los eventos cardiovasculares ${ }^{6}$. Sin embargo, en los últimos meses se conocieron resultados de estudios que obligan a replantearse al menos algunas de estas modificaciones.

El primero es un metaanálisis publicado a principios de noviembre en la revista The Lancet. El mismo comparó ensayos clínicos donde pacientes hipertensos (incluyendo pacientes con y sin enfermedad vascular o diabetes) fueron asignados a tratamiento intensivo de la TA vs tratamiento habitual o estándar. El estudio halló que el grupo de tratamiento intensivo tenía una disminución del $14 \%$ en el riesgo de tener eventos cardiovasculares mayores (esto es, IAM, ACV, falla cardiaca, o muerte cardiovascular). Por año, los efectos adversos serios fueron observados en $0,9 \%$ de los pacientes asignados a tratamiento habitual y en $1,2 \%$ de los pacientes en la rama de tratamiento intensivo. El mismo estudio encontró que el número necesario a tratar (NNT) para evitar un evento cardiovascular mayor fue 94 personas entre pacientes con antecedentes de enfermedad cardiovascular, diabetes o insuficiencia renal crónica, y 186 entre pacientes sin estos antecedentes?.

Sin embargo, la gran noticia del año en relación a este tema fue la detención temprana de la intervención del estudio SPRINT (Systolic Blood Pressure Intervention Trial) anunciada en septiembre ${ }^{8}$. SPRINT es un ensayo clínico financiado por el National Institutes of Health (NIH) de EE.UU. que aleatorizó más de 9.300 pacientes hipertensos no diabéticos mayores de 50 años con alto riesgo cardiovascular a dos ramas de tratamiento: estándar (objetivo de TAS $<140 \mathrm{mmHg}$ ) vs intensivo (objetivo de TAS $<120 \mathrm{mmHg}$ )9. La media de TAS en el grupo estándar fue de $134,6 \mathrm{mmHg}$ vs $121,5 \mathrm{mmHg}$ en el grupo intensivo. Durante el seguimiento, se encontró que en el grupo de tratamiento estándar el $2,19 \%$ de los participantes presentaba un evento cardiovascular mayor por año, mientras que en el grupo intensivo este porcentaje se reducía a 1,65\%. A lo largo

\section{Referencias}

1. Consejo Argentino de Hipertensión Arterial y Sociedad Argentina de Cardiología. Consenso de Hipertensión Arterial. Revista Argentina de Cardiologia, 2013; 81(2).

2. The National Institute for Health and Care Excellenœe (NICE), Hypertension in adults: diagnosis and management 2011. Disponible en URL h hitp://www.nice.org.uk/guidance/CG127 (Uútimo acoeso dicembre 2015).

3. James PA. y col. 2014 evidence-based guideline for the management of high blood pressure in adults: report from the panel members appointed to the Eighth Joint National Committee (JNC 8). JAMA. 2014;311(5):507-20.

4. Mancia G. y col. 2013 ESH/ESC Practice Guidelines for the Management of Arterial Hypertension. Blood Press 2014;23(1):3-16

5. Cushman WC. y col. Effects of intensive blood-pressure control in type 2 diabetes mellitus. N Engl J Med. 2010;362(17):1575-85.

6. Diao D. y col. Pharmacotherapy for mild hypertension. Cochrane Database Syst Rev. 2012;8: Cd006742.

7. Xie X. y col. Effects of intensive blood pressure lowering on cardiovascular and renal outcomes: updated systematic review and meta-analysis. Lancet. 2015 Nov 7. pii: S0140-6736(15)00805-3. doi: 10.1016/S0140-6736(15)00805-3.

8. National Institutes of Health and National Heart Lung and Blood Institute. Landmark NIH study shows intensive blood pressure management may save lives. 2015. Disponible en URL: http://www.nhlbi.nih.gov/news/press-releases/2015/landmark-nih-study-shows-intensive-blood-pressure-management-may-save-lives (último acceso diciembre 2015).

9. Wright JT. y col. A Randomized Trial of Intensive versus Standard Blood-Pressure Control. N Engl J Med. 2015;373(22):2103-16.
* Servicio de Medicina Familiar y Comunitaria del Hospital Italiano de Buenos Aires. victoria.salgado @ hospitalitaliano.org.ar 